\title{
Generalized Bowen-York Initial Data
}

\author{
R. Beig
}

Institut für Theoretische Physik, Universität Wien, Boltzmanngasse 5, A-1090 Wien, Austria

\begin{abstract}
A class of vacuum initial-data sets is described which are based on certain expressions for the extrinsic curvature first studied and employed by Bowen and York. These expressions play a role for the momentum constraint of General Relativity which is analogous to the role played by the Coulomb solution for the Gauss-law constraint of electromagnetism.
\end{abstract}

\section{Introduction}

In this lecture I wish to study a specific class of solutions to the initial-value constraints in vacuo, or, rather the 'momentum' part of these equations, which are a generalization of ones first put forward by Bowen and York ([1],[2]). While these solutions are certainly special, they turn out to be very useful. In particular, many of the initial-data sets currently used by numerical relativists are Bowen-York initial data (BY initial data), in the sense that they are based on the explicit extrinsic curvature expressions first written down in $([1],[2])$. Although we shall in this work mainly be concerned with the momentum constraints, the solutions we shall study can only be understood as ingredients to solutions to the full set of initial-value constraints.

We first recall the notion of an initial-data set (IDS). This consists of a triple $\left(\bar{\Sigma}, \bar{h}_{i j}, \bar{K}_{i j}\right)$, where $\bar{\Sigma}$ is a 3 -manifold, $\bar{h}_{i j}$ a positive-definite metric on $\bar{\Sigma}$ and $\bar{K}_{i j}$ a symmetric tensor field on $\bar{\Sigma}$. This IDS is called a vacuum IDS, if the following system of equations is satisfied

$$
\begin{aligned}
& \bar{D}^{j}\left(\bar{K}_{i j}-\bar{h}_{i j} \bar{K}\right)=0 \\
& \overline{\mathcal{R}}+\bar{K}^{2}-\bar{K}^{i j} \bar{K}_{i j}=0,
\end{aligned}
$$

where $\bar{D}_{i}$ is the Levi Civita covariant derivative associated with $\bar{h}_{i j}, \overline{\mathcal{R}}$ the scalar curvature and $\bar{K}:=\bar{h}^{i j} \bar{K}_{i j}$. Given a vacuum IDS, there is a spacetime $M$ with Ricci flat Lorentz metric $g_{\mu \nu}$, in which $\bar{\Sigma}$ is a Cauchy surface and $\bar{h}_{i j}\left(\right.$ resp. $\left.\bar{K}_{i j}\right)$ are the intrinsic metric (resp. extrinsic curvature) induced on $\bar{\Sigma}$.

We now review the 'conformal method' for solving the vacuum initialvalue constraints. This yields solutions $\left(\bar{h}_{i j}, \bar{K}_{i j}\right)$ of Equ.'s $(1,2)$ for which $\bar{K} \equiv$ const. Then, if we write

$$
\bar{K}_{i j}=\bar{\kappa}_{i j}+\frac{1}{3} \bar{h}_{i j} \bar{K}
$$


it follows that

$$
\bar{D}^{j} \bar{\kappa}_{i j}=0 \quad \text { and } \quad \bar{\kappa}=\bar{\kappa}_{i j} \bar{h}^{i j}=0 .
$$

Thus $\bar{\kappa}_{i j}$ is a "transverse-tracefree" (TT-)tensor. The conformal method rests on two identities.

Fact a): Let $h_{i j}$ and $\bar{h}_{i j}$ be two conformally related metrics, i.e.

$$
\bar{h}_{i j}=\phi^{4} h_{i j}, \quad \phi>0 .
$$

Then, defining the conformal Laplacian $L_{h}$ to be

$$
L_{h}:=-h^{i j} D_{i} D_{j}+\frac{1}{8} \mathcal{R}[h]=-\Delta+\frac{1}{8} \mathcal{R}
$$

we have that

$$
L_{\bar{h}}\left(\phi^{-1} \psi\right)=\phi^{-5} L_{h} \psi, \quad \phi>0 .
$$

Setting in (7) $\phi=\psi$, it follows that

$$
L_{\bar{h}} 1=\frac{1}{8} \mathcal{R}[\bar{h}]=\phi^{-5} L_{h} \phi .
$$

Fact b): Suppose $K_{i j}=K_{(i j)}$ is trace-free. Then

$$
\bar{D}^{j} \bar{K}_{i j}=\phi^{-6} D^{j} K_{i j}, \quad \phi>0,
$$

where $\bar{K}_{i j}=\phi^{-2} K_{i j}$. Combining these facts we can make the following observation: Suppose $K_{i j}$ is a TT-tensor with respect to the metric $h_{i j}$. Then, for any $\phi>0$ and any constant $\bar{K}$,

$$
\bar{K}_{i j}=\phi^{-2} K_{i j}+\frac{\bar{K}}{3} \phi^{2} h_{i j}
$$

satisfies Equ. (1). Furthermore, if $\phi$ satisfies

$$
L_{h} \phi=\frac{1}{8} K_{i j} K^{i j} \phi^{-7}-\frac{1}{12} \bar{K}^{2} \phi^{5}
$$

then $\bar{h}_{i j}=\phi^{4} h_{i j}$ satisfies Equ. (2). Thus, solving the constraints $(1,2)$ amounts to choosing a 'background metric' $h_{i j}$, finding a TT-tensor $K_{i j}$ with respect to $h_{i j}$ and solving Equ. (11) for given $\left(h_{i j}, K_{i j}\right)$ and a choice of constant $\bar{K}$.

We will need global conditions in order for the above program to go through. The two cases of greatest interest is, firstly, the case where $\bar{\Sigma}$ is compact ("cosmological case"), and here it is natural to take the background fields $\left(h_{i j}, K_{i j}\right)$ to be defined on $\Sigma=\bar{\Sigma}$. Depending on the global conformal nature of $h_{i j}$, the sign of $\bar{K}$ and on whether $\bar{\kappa}_{i j}$ is zero or non-zero, there is an exhaustive list of possibilities [3] for which Equ. (11) can be solved. 
The other case is that where $\left(\bar{\Sigma}, \bar{h}_{i j}, \bar{K}_{i j}\right)$ is asymptotically flat. This means that there is a compact subset $K \subset \bar{\Sigma}$, so that $\bar{\Sigma} \backslash K$ consists of a finite number of asymptotic ends. An asymptotic end is a set diffeomorphic to $\mathbf{R}^{3} \backslash B$, where $B$ is a closed ball. Furthermore, in the coordinate chart given by this diffeomorphism, $\bar{h}_{i j}$ should satisfy

$$
\begin{gathered}
\bar{h}_{i j}-\delta_{i j}=O\left(\frac{1}{\bar{r}}\right), \quad \bar{r}^{2}=\bar{x}^{i} \bar{x}^{j} \delta_{i j}, \\
\bar{K}_{i j}=O\left(\frac{1}{\bar{r}^{2}}\right)
\end{gathered}
$$

and $\partial \bar{h}_{i j}=o\left(1 / \bar{r}^{2}\right), \ldots, \partial \bar{K}_{i j}=O\left(1 / \bar{r}^{3}\right), \ldots$ for a few derivatives. Since we require $\bar{K}=$ const, it follows that $\bar{K}=0$.

Now the problem of solving the constraints takes the following form: One first picks an asymptotically flat 3 -metric $h_{i j}$ on $\bar{\Sigma}$ and a TT-tensor $K_{i j}$ on $\left(\bar{\Sigma}, h_{i j}\right)$. One then has to solve Equ. (11) with $\bar{K}=0$ and the boundary condition that $\phi \rightarrow 1$ at infinity.

A very convenient alternative is to take $\left(h_{i j}, K_{i j}\right)$ to be defined on a compact manifold $\Sigma$, the many-point compactification of $\bar{\Sigma}$. The "infinities" of $\bar{\Sigma}$ are now replaced by a finite number of points $\Lambda_{\alpha} \in \Sigma$, which we call punctures. The Equ. (11) should then be replaced by

$$
L_{h} \phi=\frac{1}{8} K_{i j} K^{i j} \phi^{-7}+4 \pi\left(c_{1} \delta_{1}+c_{2} \delta_{2}+\ldots\right),
$$

where $\delta_{\alpha}$ is the delta distribution supported at $\Lambda_{\alpha}$ and $c_{\alpha}$ are positive constants. Note that $\bar{K}=0$. Let $h_{i j}$ be a smooth metric on $\Sigma$ and $K_{i j}$ be smooth on $\Sigma$ except, perhaps, at the points $\Sigma_{\alpha}$, where it may blow up like $1 / r^{4}$, where $r^{2}=\delta_{i j} x^{i} x^{j}$ with $x^{j}$ being Riemann normal coordinates centered at $\Lambda_{\alpha}$. Then a solution $\phi$ of Equ. (14) behaves like $1 / r$ near $\Lambda_{\alpha}$. If a positive global solution of Equ. (14) exists, then $\bar{h}_{i j}=\phi^{4} h_{i j}, \bar{K}_{i j}=\phi^{-2} K_{i j}$ satisfy the conditions of asymptotic flatness in the "inverted" coordinates $\bar{x}^{i}=x^{i} / r^{2}$. For the conditions under which $\phi>0$ exists, see e.g. [4].

\section{The Gauss law constraint of electromagnetism}

Before turning to the methods for obtaining TT-tensors, it is very instructive to use, by means of analogy, the equation

$$
\operatorname{div} E=D^{i} E_{i}=0
$$

on a Riemann 3-manifold $\left(\Sigma, h_{i j}\right)$, that-is-to-say the Gauss constraint of electrodynamics. This, like the TT-condition, is an underdetermined elliptic system. This means that the "symbol map", namely the linear map $\sigma(k)$, sending

$$
e_{i} \in \mathbf{R}^{3} \mapsto k^{i} e_{i} \in \mathbf{R} \quad\left(k \in \mathbf{R}^{3}, k \neq 0\right)
$$


is onto. (The symbol map is essentially the Fourier transform of the highestderivative term of a partial differential operator.) Note, first of all, that, when $\bar{h}_{i j}=\phi^{4} h_{i j}(\phi>0), \bar{E}_{i}=\phi^{-2} E_{i}$, we have that

$$
\bar{D}^{i} \bar{E}_{i}=\phi^{-6} D^{i} E_{i} .
$$

We will thus again take $\Sigma$ to be compact, imagining that we are either in the cosmological case or that $\Sigma$ is the conformally compactified, asymptotically flat 3-space $\bar{\Sigma}$. Our first point is to show two different ways of solving (15). To describe the first one, which is the analogue of the "York method" for solving the momentum constraint, we observe that the gradient operator grad, sending $C^{\infty}$-functions on $\Sigma$ to $\Lambda^{1}(\Sigma)$, the 1 -forms on $\Sigma$, is (minus) the formal adjoint of div under the natural $L^{2}$-inner product on $\left(\Sigma, h_{i j}\right)$. It is thus, on general grounds (see Appendix on Sobolev Spaces and Elliptic Operators in [5]) true that there is a direct-sum decomposition of $\Lambda^{1}(\Sigma)$ as

$$
\Lambda^{1}(\Sigma)=\operatorname{grad}\left(C^{\infty}(\Sigma)\right) \oplus \text { ker div }
$$

and this decomposition is orthogonal in the $L^{2}$-sense. Furthermore the second summand in (18) is infinite-dimensional. The relation (18) tells us how to find the elements of ker div, i.e. the solutions of Equ. (15). Namely, pick an arbitrary 1 -form $\omega_{i}$ and write

$$
\omega_{i}=D_{i} \varphi+E_{i},
$$

where $E_{i} \in$ ker div. Hence

$$
D^{i} \omega_{i}=\operatorname{div} \operatorname{grad} \varphi=\Delta \varphi .
$$

Since the left-hand side of (20) is a divergence, it is orthogonal to ker $\Delta$. Thus $\Delta^{-1} \operatorname{div} \omega$ exists. Consequently, $E_{i}$ can be written as

$$
E=\left[\mathbf{1}-\operatorname{grad}(\operatorname{div} \operatorname{grad})^{-1} \operatorname{div}\right] \omega,
$$

and this is the general solution of (15). The Equ. (21) is a special case of the way to solve an underdetermined elliptic system. It has the feature that it is non-local, since it involves the operation of taking the inverse of div grad.

There is another way of solving Equ. (15). Observe that any 1-form $E_{i}$ of the form $\left(\varepsilon_{i j k}\right.$ is the volume element on $\left.\Sigma\right)$

$$
E_{i}=\varepsilon_{i}{ }^{j k} D_{j} \mu_{k},
$$

or

$$
E=\operatorname{rot} \mu
$$

solves Equ. (15). Is this the general solution? The answer is: yes, up to at most a finite-dimensional space, namely the harmonic 1-forms, i.e. the elements 
of $\Lambda^{1}(\Sigma)$ which are annihilated by $\Delta_{H}=(\operatorname{rot})^{2}-\operatorname{grad}$ div. More specifically we have

$$
\operatorname{ker} \operatorname{div}=\operatorname{ker} \Delta_{H} \oplus \operatorname{rot}\left(\Lambda^{1}(\Sigma)\right) .
$$

Note that $\operatorname{ker} \Delta_{H}=$ ker $\operatorname{rot} \cap$ ker div. The relation (23) gives a refinement of the decomposition (20). Stated in a more fancy way, the decomposition (23) expresses the fact that the de Rham cohomology group $H^{2}=$ ker $\operatorname{div} / \operatorname{rot} \Lambda^{1}(\Sigma)$ is isomorphic to ker $\Delta_{H}$. Let us for simplicity assume that ker $\Delta_{H}$ is trivial. In Wheeler's words [6] we assume that there is no "charge without charge". This will for example be the case when $\left(\Sigma, h_{i j}\right)$ is the standard 3-sphere. More generally, this is the case when $\left(\Sigma, h_{i j}\right)$ is of constant positive curvature (Exercise: prove this). This implies the following: Let $E_{i}$ be an arbitrary solution of Equ. (15), and $S \subset \Sigma$ be an arbitrary embedded 2 -sphere. Then the integral $\int_{S} E_{i} d S^{i}$ is zero, i.e.

$$
\int_{S} E_{i} d S^{i}=0
$$

Suppose now that $\Sigma$ contains some punctures, at which we are willing to allow $D^{i} E_{i}$ to become singular. Then Equ. (24) will in general no longer be valid, and $E=\operatorname{rot} \mu$ will no longer be the general solution to Equ. (15). Suppose for simplicity that $\Sigma$ is diffeomorphic to the 3 -sphere. If there is just one puncture $\Lambda_{1}$ (not to be confused with $\Lambda^{1}(\Sigma)$ !), then (24) is clearly still valid, whether $S$ endores $\Lambda_{1}$ or it doesn't. But suppose there are two punctures, $\Lambda_{1}$ and $\Lambda_{2}$. Then, either $S$ does not enclose either of them in which case (24) is valid or it does, in which case (24) can not be expected to hold in general. The value

$$
Q=\int_{S} E_{i} d S^{i}
$$

in the latter case, constitutes a 1-parameter class of obstructions to the existence of $\mu_{i}$ such that $E=\operatorname{rot} \mu$. (Of course, $Q$ depends only on the homology class of $S$, not $S$ itself.) It is thus desirable to split the general $E$ with $\operatorname{div} E=0$ as a sum of one of the form $E=\operatorname{rot} \mu$ and a set of fields parametrized by $Q$. Imagine this latter set of fields to be distributions which are orthogonal to fields in the first summand. Thus these will be of the form $E=\operatorname{grad} \varphi$, and

$$
\operatorname{div} \operatorname{grad} \varphi=\rho,
$$

where the distribution $\rho$ is supported on $\Lambda_{1} \cup \Lambda_{2}$ and has to satisfy

$$
\int_{\Sigma} \rho d V=0
$$

Thus we set

$$
\rho=Q\left(\delta_{1}-\delta_{2}\right)
$$


where $\delta$ is the Dirac delta distribution. If $\varphi$ is the solution (unique up to addition of a constant) of Equ. (27), $E=\operatorname{grad} \varphi$ and the normal to $S$ points towards $\Lambda_{2}$, then Equ. (25) is fulfilled.

Let us be more specific. When $\left(\Sigma, h_{i j}\right)$ is the standard 3 -sphere, it can be imagined to be the 1-point conformal compactification (conformal compactification by inverse stereographic projection from the origin, say) of flat $\mathbf{R}^{3}=\bar{\Sigma}$ (which in turn can be viewed to be a standard $t=$ const hyperplane of the Minkowski spacetime of Special Relativity). Suppose the origin of $\bar{\Sigma}$ corresponds to $\Lambda_{1}$ and $\Lambda_{2}$ is the antipode of $\Lambda_{1}$ on $S^{3}$. Thus $\Lambda_{1}$ should be viewed as a point where the field becomes singular and $\Lambda_{2}$ as the point-atinfinity. (In the gravity case, of course, singularities do not make good sense, sp all punctures will play the role of points-at-infinity. That this is possible, is due to the fact that $\bar{h}_{i j}$ on $\bar{\Sigma}$ is not a fixed element of the theory, but $\bar{h}_{i j}=\phi^{4} h_{i j}$, and $\phi$ satisfies Equ. (14).) We now undo the stereographic projection and write

$$
\bar{E}_{i}=\phi^{-2} D_{i} \varphi,
$$

where $\varphi$ solves (26) and $\phi$ solves

$$
L_{h} \phi=4 \pi \delta_{1} .
$$

The resulting field $\bar{E}_{i}$ is nothing but the Coulomb field on $\bar{\Sigma}=\mathbf{R}^{3}$ with charge $Q$ sitting at the origin.

The above situation can be slightly generalized. Let $\rho$ be an arbitrary distribution on $\Sigma$ such that

$$
\int_{\Sigma} \rho d V=0
$$

In particular $\rho$ could be a smooth function. Then, if there are no harmonic 1-forms, the general solution of

$$
D^{i} E_{i}=\rho
$$

is of the form

$$
E=\operatorname{rot} \mu+\operatorname{grad} \varphi
$$

where

$$
\operatorname{div} \operatorname{grad} \varphi=\rho .
$$

The second term in Equ. (33) could be called the generalized Coulomb field corresponding to the source $\rho$ or, alternatively, the "longitudinal" solution of Equ. (32).

\section{The momentum constraint}

We will now turn to the gravity case, i.e. the momentum constraints. The solutions originally due to Bowen and York (resp. our generalization thereof) 
will turn out to be close analogues of the Coulomb field (resp. the generalized Coulomb field), as described above. When $\left(\Sigma, h_{i j}\right)$ is again taken to be standard $S^{3}$ with antipodal punctures $\Lambda_{1}, \Lambda_{2}$ there will be a 10-parameter set of sources for the TT-condition $D^{j} K_{i j}=0, K=0$, so that, under stereographic projection relative to $\Lambda_{1}$, the TT-tensor on punctured $\mathbf{R}^{3}$ corresponding to the longitudinal solution of the inhomogeneous TT-condition on $\Sigma$ contain exactly the ones written down by Bowen and York. How does the number 10 enter here? In the Maxwell case we had 1, which was the null space of grad, which in turn is the adjoint of the operator div. In the gravity case 10 arises as the null space of the adjoint to the operator div, acting on symmetric, trace-free tensors, namely the conformal Killing operator. This null space, in turn, is the space of conformal Killing vectors, which has at most 10 dimensions on a 3 -manifold $\Sigma$. We now have to explain these things in more detail. We again take $\left(\Sigma, h_{i j}\right)$ to be compact. The underdetermined elliptic system

$$
(\operatorname{div} K)_{i}=D^{j} K_{i j}=0, \quad K=0
$$

is, in complete analogy with the Maxwell case, solved by the "York decomposition"

$$
Q_{i j}=(L W)_{i j}+K_{i j}
$$

where $Q_{i j}$ is an arbitrary symmetric, trace-free tensor on $\left(\Sigma, h_{i j}\right)$ and $L$ is the conformal Killing operator

$$
(L W)_{i j}:=D_{i} W_{j}+D_{j} W_{i}-\frac{1}{3} h_{i j} D^{\ell} W_{\ell},
$$

(not to be confused with the conformal Laplacian $L_{h}$ ) which is (-1/2 times) the adjoint of div. Hence, in order for $K_{i j}$ to be in the null space of div, we have that

$$
\operatorname{div} \circ L W=\operatorname{div} Q .
$$

The operator on the left in Equ. (38) is elliptic with null space consisting of conformal Killing vectors (Proof: Contract with a covector $\lambda$ and integrate by parts!). Thus, in complete analogy to Equ. (21) in the electromagnetic case (15), the general solution $K_{i j}$ of the TT-condition, Equ. (35), is given by

$$
K=\left[\mathbf{1}-L(\operatorname{div} \circ L)^{-1} \operatorname{div}\right] Q,
$$

where $Q_{i j}$ is an arbitrary symmetric, trace-free tensor. The above procedure works for an arbitrary $\left(\Sigma, h_{i j}\right)$ with $\Sigma$ compact. It would also work if $\Sigma$ was asymptotically flat (see e.g. [7]). We now again ask the question as to whether there is a more explicit method for finding TT-tensors where (35) is solved "by differentiation" rather than "by integration", as in Equ. (39). There is a positive answer, but only in the case where $\left(\Sigma, h_{i j}\right)$ is (locally) conformally flat. Luckily, this comprises many of the IDS's which are currently in use by the numerical relativists, as we shall describe in Sect. 5. The necessary and 
sufficient condition [8] for $h_{i j}$ to be conformally flat is that the Cotton-York tensor $\mathcal{H}_{i j}$ defined by

$$
\mathcal{H}_{i j}=\varepsilon_{k \ell(i} D^{k} \mathcal{R}^{\ell}{ }_{j)}
$$

be zero. Note that $\mathcal{H}_{i j}$ is always symmetric, trace-free. Also, as a consequence of the Bianchi identities, it is divergence-free. Thus $\mathcal{H}_{i j}$ is a TT-tensor with respect to $h_{i j}$. If $h_{i j}$ is such that $\mathcal{H}_{i j}$ vanishes, it follows that the operator $H_{i j}$, obtained by linearization of $\mathcal{H}_{i j}$ at $h_{i j}$, is a (third-order) partial differential operator mapping symmetric tensors $\ell_{i j}$ (we take them also to be trace-free) into tensors which are TT with respect to $h_{i j}$. We refrain here from writing down the operator $H_{i j}$ explicitly (see [9]). There now arises the question of whether $K_{i j}$ given by $K_{i j}=H_{i j}(\ell)$ is the general TT-tensor. The answer, given in [9], is again "yes" up to a finite dimensional set of "harmonic TTtensors", i.e. symmetric, trace-free tensors $\ell_{i j}$ which satisfy both $D^{j} \ell_{i j}=0$ and $H_{i j}(\ell)=0$. What is the condition for the absence of such harmonic TT-tensors? In allusion to Wheeler [6] we might describe this situation by the absence of "momentum without momentum". It is shown in [9], that this condition is exactly that, for any 2 -surface $S$ embedded in $\Sigma$ and any conformal Killing vector $(\mathrm{CKV}) \xi^{i}$ on $\left(\Sigma, h_{i j}\right)$ which is defined near $S$, there holds

$$
\int_{S} K_{i j} \xi^{i} d S^{j}=0
$$

for all TT-tensors $K_{i j}$. We now come to the inhomogeneous equation

$$
D^{\ell} K_{i \ell}=j_{i}
$$

which is the analogue of the Maxwell equation (32). It would be nice to have a very clear geometrical motivation for our expression for $j_{i}$, but we have to leave that for future work. The proposal is that $j_{i}$ depends on a "charge density $\rho "$ and a CKV $\eta^{i}$, as follows:

$j_{i}(\eta)=-D^{\ell}\left(D_{[\ell} \eta_{i]} \rho\right)+\frac{2}{3}\left(D_{i} D_{\ell} \eta^{\ell}\right) \rho+\frac{2}{3} D_{i} D_{\ell}\left(\eta^{\ell} \rho\right)+\frac{2}{9} D_{i}\left(\left(D_{\ell} \eta^{\ell}\right) \rho\right)+4 L_{i \ell} \eta^{\ell} \rho$,

where $L_{i j}=\mathcal{R}_{i j}-\frac{1}{4} h_{i j} \mathcal{R}$. It is important that Equ. (42) behaves naturally under conformal rescalings of the metric, i.e. $\bar{h}_{i j}=\phi^{4} h_{i j}$. This is the case since one can show that

$$
\bar{\jmath}_{i}(\eta)=\omega^{-6} j_{i}(\eta) .
$$

Thus, with $\bar{K}_{i j}=\omega^{-2} K_{i j}, \bar{K}_{i j}$ satisfies $\bar{D}^{\ell} \bar{K}_{i \ell}=\bar{\jmath}_{i}$ provided $K_{i j}$ solves Equ. (42).

Suppose we have an open region $\Omega \subset \Sigma$, bounded by $S$. Then it follows from (43) that

$$
\int_{S} K_{i j} \lambda^{i} d S^{j}=\int_{\Omega} X(\lambda, \eta) \rho d V
$$


where

$$
X(\lambda, \eta)=D^{[i} \lambda^{j]} D_{[i} \eta_{j]}+\frac{2}{3}\left[\lambda^{i} D_{i} D_{j} \eta^{j}+\eta^{i} D_{i} D_{j} \lambda^{j}\right]-\frac{2}{9}\left(D_{i} \lambda^{i}\right)\left(D_{j} \eta^{j}\right)+4 L_{i j} \lambda^{i} \eta^{j}
$$

The bilinear form $X$ has an important geometrical meaning. Recall that the CKV's span a vector space $\mathbf{W}$ of at most ten dimensions. (The dimension ten is reached when $\Sigma$ is simply connected, in which case the general CKV $\eta^{i}$ can be characterized by the values of $\eta^{i}$ at some point $p \in \Sigma$, together with those of $D_{[i} \eta_{j]}, D_{j} \eta^{j}$ and $D_{i} D_{j} \eta^{j}$, the so-called "conformal Killing data" [10].) The Lie commutator of vector fields on $\Sigma$ induces a Lie algebra structure on $\mathbf{W}$. The form $X(\lambda, \eta)$, for $\eta$ and $\lambda$ both CKV's, is nothing but $(1 / 3)$ times the Killing metric (see App. B of [11]). One can check by explicit computation that

$$
X(\lambda, \eta)=\text { constant on } \Sigma,
$$

when $\lambda$ and $\eta$ are both CKV's. Thus, from (45)

$$
\int_{S} K_{i j} \lambda^{i} d S^{j}=X(\lambda, \eta) \int_{\Omega} \rho d V .
$$

So, similar to the Maxwell case, $\rho$ can not in general be a distribution concentrated at a single point. Rather, when $\Omega=\Sigma$ it follows that

$$
X(\lambda, \eta) \int_{\Sigma} \rho d V=0 .
$$

Equ. (50) is the necessary and sufficient condition in order for Equ. (42), with $j_{i}$ given by (43), to have solutions. Note that, when $\operatorname{dim} \mathbf{W}$ is 10 , the Killing metric is non-degenerate. The solution of the equation

$$
D^{\ell} K_{i \ell}=j_{i}(\eta)
$$

becomes unique when we require $K_{i j}$ to be longitudinal, i.e.

$$
K_{i j}=(L W)_{i j}
$$

It remains to solve the elliptic equation

$$
\operatorname{div} \circ L W=j(\eta)
$$

The most important case is again where $\left(\Sigma, h_{i j}\right)$ is the standard three-sphere and

$$
\rho=-2 \pi\left(\delta_{1}-\delta_{2}\right) \text {. }
$$

To write down explicitly the 10-parameter set of solutions, it is convenient to send $\Lambda_{2}$ to infinity by a stereographic projection. Then we have again Equ. 
(49), but on $\left(\mathbf{R}^{3}, \delta_{i j}\right)$, punctured at the origin. The CKV's on $\mathbf{R}^{3}$ fall into the following classes

$$
\begin{aligned}
& { }^{1} \eta^{i}(x)=Q^{i}, \quad Q^{i}=\mathrm{const} \\
& { }^{2} \eta^{i}(x)=\varepsilon^{i}{ }_{j k} S^{j} x^{k}, \quad S^{i}=\mathrm{const} \\
& { }^{3} \eta^{i}(x)=C x^{i}, \quad C=\mathrm{const} \\
& { }^{4} \eta^{i}(x)=(x, x) P^{i}-2(x, P) x^{i}, \quad P^{i}=\text { const. }
\end{aligned}
$$

Here $x^{i}$ are cartesian coordinates. We find

$$
\begin{aligned}
{ }^{1} K_{i j}(x) & =\frac{3}{2 r^{2}}\left[P_{i} n_{j}+P_{j} n_{i}-\left(\delta_{i j}-n_{i} n_{j}\right)(P, n)\right] \\
{ }^{2} K_{i j}(x) & \left.=\frac{6}{r^{3}} \varepsilon_{k \ell(i} S^{k} n^{\ell} n_{j}\right) \\
{ }^{3} K_{i j}(x) & =\frac{C}{r^{3}}\left(3 n_{i} n_{j}-\delta_{i j}\right) \\
{ }^{4} K_{i j}(x) & =\frac{3}{2 r^{4}}\left[-Q_{i} n_{j}-Q_{j} n_{i}-\left(\delta_{i j}-5 n_{i} n_{j}\right)(Q, n)\right] .
\end{aligned}
$$

Here $n_{i}=x_{i} / r$. The constants $P_{i}$ in (58) play the role of the linear ADMmomentum at $r=\infty$ and the $S^{i}$ in (59) are the ADM angular momentum. Thus $P_{i}$ and $S_{i}$ are conserved under time evolution. If we had sent the point $\Lambda_{1}$ to infinity, or - what is the same - if we made an inversion of the form $\bar{x}^{i}=x^{i} / a^{2} r^{2}$ ("Kelvin transform"), the $Q_{i}$ would be $a^{2}$ times the linear momentum at $r=0$, viewed as another infinity. Similarly (54) goes over into (57) with $P^{i}=Q^{i}$ under Kelvin transform. The role of the constant $C$ is less clear. It was used in [4], to construct IDS's which have future-trapped surfaces. The constant $C$ does not correspond to a conserved quantity.

If $K_{i j}$ was a sum of the expression in (58) and that in (59) the physical interpretation is that they characterize a single black hole with momentum $P^{i}$ and spin $S^{i}$.

We now come to the technical result of this section. Suppose $\left(\Sigma, h_{i j}\right)$ is of constant curvature, i.e.

$$
\mathcal{R}_{i j k \ell}=\frac{\mathcal{R}}{3} h_{k[i} h_{j] \ell}, \quad \mathcal{R}=\text { const. }
$$

Suppose, further, we know a function (distribution) $G$ satisfying

$$
\Delta G=\rho .
$$

In the case when $\Sigma$ is compact, this will exist provided that $\int_{\Sigma} \rho d V=0$. Then there is an explicit expression for $W_{i}$ solving Equ. (52), namely

$$
\begin{aligned}
W_{i} & =\frac{1}{2} \eta^{j} D_{j} D_{i} G+\frac{3}{2}\left(D_{i} \eta_{j}\right) D^{j} G+\left(D_{j} \eta^{j}\right) D_{i} G+\frac{\mathcal{R}}{3} \eta_{i} G \\
& =-D^{j}\left[\left(D_{[j} \eta_{i]}\right) G\right]-\frac{1}{6} D_{i}\left[\left(D_{j} \eta^{j}\right) G\right]+\frac{1}{2} D_{i} D_{j}\left(\eta^{j} G\right) .
\end{aligned}
$$


For an outline of the proof, see [12]. It is easy to check that, when $\Sigma$ is flat $\mathbf{R}^{3}, \rho=-2 \pi \delta(x)$ and $\xi^{i}$ runs through (54-57), the $K_{i j}$ 's given by $(L W)_{i j}$ with $W_{i}$ as in Equ. (64), reduce to (58-61).

\section{Boosting a single black hole}

In this section we present a simple perturbative calculation which should serve as a check whether the "boost-type" extrinsic curvature (58) gives a sensible result for the full IDS. We assume that $\left(\Sigma, h_{i j}\right)$ is a standard threesphere and we try to solve the Lichnerowicz equation (14) with $\Lambda_{1}$ and $\Lambda_{2}$ being south and north pole, respectively. The $K_{i j}$ in (14) should be the one turning into (58) after stereographic projection. When $K_{i j}$ vanishes and the three-sphere has radius $1 / m$, the unique solution to (14) gives rise to the Schwarzschild solution of mass $m$. After stereographic projection the threesphere punctured at $\Lambda_{1}$ and $\Lambda_{2}$ becomes flat $\mathbf{R}^{3}$, punctured at the origin. The Lichnerowicz conformal factor $\phi$ such that $\bar{h}_{i j}=\phi^{4} \delta_{i j}$ for Schwarzschild is, as is well-known,

$$
\phi=1+\frac{m}{2 r} .
$$

By the conformal invariance of the York procedure we can of course also start from flat, punctured $\mathbf{R}^{3}$. We are thus trying to solve

$$
\Delta \phi=-\frac{1}{8} K_{i j} K^{i j} \phi^{-7}
$$

with $K_{i j}$ given by Equ. (58), and $\phi$ should go to one at infinity and have a $1 / r$-singularity near $r=0$ so that $\phi^{4} \delta_{i j}$ becomes asymptotically flat both near infinity and near $r=0$. We have that

$$
K_{i j} K^{i j}=\frac{9}{2 r^{4}}\left[P_{i} P^{i}+2\left(P_{i} n^{i}\right)^{2}\right] .
$$

For $\phi$ we make the ansatz

$$
\phi=1+\frac{m}{2 r}+\psi,
$$

where $\psi$ should vanish at $r=\infty$ and be regular near $r=0$. We only keep terms quadratic in $P_{i}$. It follows that

$$
\Delta \psi=-\frac{9 r^{3}}{16} \frac{P^{2}+2(P, n)^{2}}{(r+m / 2)^{7}}
$$

Thus

$$
\psi(x)=\frac{9}{4 \pi \cdot 16} \int_{\mathbf{R}^{3}} \frac{r^{\prime 3}}{\left|x-x^{\prime}\right|} \frac{P^{2}+2\left(P, n^{\prime}\right)^{2}}{\left(r^{\prime}+m / 2\right)^{7}} d x^{\prime} .
$$

It is not difficult to find that

$$
\lim _{x \rightarrow 0} \psi(x)=\frac{P^{2}}{8 m^{2}}
$$


and

$$
\psi(x)=\frac{5 P^{2}}{16 m r}+O\left(\frac{1}{r^{2}}\right),
$$

near $r=\infty$. While the constant $m$ is only a formal parameter, the true "observables" are the ADM-energies at the two infinities. The ADM energy $M$ at $r=\infty$ is given by

$$
M=m+\frac{5 P^{2}}{8 m} \text {. }
$$

The ADM energy $\bar{M}$ near $r=0$ is obtained by noting that, near $r=0$,

$$
d \bar{s}^{2}=\bar{h}_{i j} d x^{i} d x^{j}=\left[1+\frac{P^{2}}{8 m^{2}}+\frac{m}{2 r}+O(r)\right]^{4} \delta_{i j} d x^{i} d x^{j} .
$$

After inversion $\bar{x}^{i}=(m / 2)^{2} x^{i} / r^{2}$, this results in

$$
\begin{aligned}
d \bar{s}^{2} & =\left[1+\frac{P^{2}}{8 m^{2}}+\frac{2 \bar{r}}{m}+O\left(\frac{1}{\bar{r}}\right)\right]^{4}\left(\frac{m}{2}\right)^{4} \frac{1}{\bar{r}^{4}} \delta_{i j} d \bar{x}^{i} d \bar{x}^{j} \\
& =\left[\frac{m}{2 \bar{r}}\left(1+\frac{P^{2}}{8 m^{2}}\right)+1+O\left(\frac{1}{\bar{r}^{2}}\right)\right] \delta_{i j} d \bar{x}^{i} d \bar{x}^{j} .
\end{aligned}
$$

Consequently,

$$
\bar{M}=m+\frac{P^{2}}{8 m}+O\left(P^{4}\right) .
$$

Thus,

$$
m=\bar{M}-\frac{P^{2}}{8 \bar{M}}+O\left(P^{4}\right) .
$$

Inserting (77) into (73) we finally obtain

$$
M=\bar{M}+\frac{P^{2}}{2 \bar{M}}+O\left(P^{4}\right)
$$

or

$$
M^{2}-P^{2}=\bar{M}^{2}+O\left(P^{4}\right) .
$$

Recall that with our ansatz for $K_{i j}$ an observer near $r=\infty$ "sees" a hole at $r=0$ with momentum $P$ and one easily finds that an observer near $r=0$ sees a hole at $r=\infty$ at momentum zero. Thus Equ. (79) says that the rest-masses at both asymptotic ends are equal. This equality expresses the "absence of gravitational radiation" on the spacetime slice $\Sigma$. If there was more than one hole, the analogue of Equ. (79) would contain for example potential energy-contributions from the mutual gravitational interaction between those holes, as in the famous calculation by Brill and Lindquist [13]. See [14] for interaction energies of more general geometries. Had we used as our ansatz for $K_{i j}$ a sum of the one in Equ. (58) and the one in (61) we would obtain

$$
M+\frac{\bar{P}^{2}}{2 M}+O\left(\bar{P}^{4}\right)=\bar{M}+\frac{P^{2}}{2 \bar{M}}+O\left(P^{4}\right),
$$


where $\bar{P}$ is the ADM 3-momentum of the $r=0$-end of $\Sigma$, which is related to $Q$ by $\bar{P}=4 Q / M^{2}$. I thank U.Kiermayr for performing this computation.

Does, for the present IDS, the relation

$$
M^{2}-P^{2}=\bar{M}^{2}-\bar{P}^{2}
$$

hold exactly? In this connection we should point out that the present data is not the same as the one induced by the Schwarzschild spacetime of mass $M$ on a boosted maximal slice, since the metric on such slices can not be conformally flat (see [15]).

\section{More general initial-data sets with punctures}

Let again $\left(\Sigma, h_{i j}\right)$ be a compact, conformally flat manifold and $\eta^{i}$ a CKV on $\Sigma$. Then consider the equation (50), namely

$$
D^{\ell} K_{i \ell}=j_{i}(\eta),
$$

with $j_{i}(\eta)$ given by Equ. (43). Although we assumed in our heuristic discussion that there are no "harmonic" TT-tensors on $\Sigma$, this is actually not required for (81) to make sense. The simplest case is where $\Sigma$ is a standard three-sphere and $\rho$ is a delta function concentrated at a finite number of points $\Lambda_{\alpha} \in \Sigma(\alpha=1, \ldots, N)$. We have to have

$$
\int_{\Sigma} \rho d V=0
$$

so that $N \geq 2$. The case $N=2$ contains the situation discussed in the previous section. The case of general $N$ is for some choices of $\eta^{i}$ leads to the IDS's studied by Brandt and Brügmann [16].

Another interesting case is that where $\left(\Sigma, h_{i j}\right)$ is $S^{2} \times S^{1}(a)$, i.e. the unittwo sphere times the circle of length $a$. This is conformally flat but not of constant curvature. The equation

$$
L_{h} \phi=4 \pi \delta_{1}
$$

has a unique positive solution $\phi$ (since $\mathcal{R}>0$, see [17]). The manifold $(\bar{\Sigma}=$ $\Sigma \backslash \Lambda_{1}, \bar{h}_{i j}$ ) with $\bar{h}_{i j}=\phi^{4} h_{i j}$ is nothing but the (time-symmetric) Misner wormhole [18]. Taking two punctures $\Lambda_{1}$ and $\Lambda_{2}$ on $\Sigma$ at the same location in $S^{2}$ and at opposite points in $S^{1}(a)$ and solving

$$
L_{h} \phi=4 \pi\left(c_{1} \delta_{1}+c_{2} \delta_{2}\right),
$$

(which can be done by linearly superposing two solutions like that in (84)) we get for $\left(\bar{\Sigma}, \bar{h}_{i j}\right)$ two asymptotically flat sheets joined by two Einstein-Rosen 
bridges [19]. These two time-symmetric IDS's can be turned into generalized Bowen-York ones by first solving

$$
D^{\ell} K_{i \ell}=j_{i}(\eta)
$$

for some CKV $\eta^{i}$ and then solving

$$
L_{h} \phi=\frac{1}{8} K_{i j} K^{i j} \phi^{-7}+4 \pi \sum_{\alpha} c_{\alpha} \delta_{\alpha} .
$$

We just deal with Equ. (86) here. In the first case (1 puncture) we cannot have $\int \rho=0$, so that $\eta$ has to be in the null space of $X(\xi, \eta)$, where $\xi$ runs through all CKV's on $\left(\Sigma, h_{i j}\right)$. But, on this manifold, all conformal Killing vectors are in fact Killing vectors. (This would be true for any compact Riemannian manifold except standard $S^{3}$, see [20] or also App. A of [21].) Hence the CKV's just comprise rotations in the $S^{2}$-direction and a covariant constant vector in the $S^{1}$-direction. The latter, from Equ. (45), is allowed, but not the rotations. Thus, within the method of this paper, it is possible to boost a Misner wormhole into the direction connecting the two wormhole throats, but it is impossible to spin up a Misner wormhole. In the Einstein-Rosen case both options are available (see Bowen, York [22], Kulkarni et al. [23] and Bowen et al. [24]). It is not clear to me whether the solutions of (81) constructed by these authors, when viewed as ones on the handle manifold $S^{2} \times S^{1}(a)$, are longitudinal or not.

\section{Acknowledgement}

This work was supported by Fonds zur Förderung der wissenschaftlichen Forschung in Österreich, Projekt Nr. P12626-PHY. I thank U.Kiermayr for a discussion which led to the discovery of an error in a previous version of this work.

\section{References}

1. Bowen J M 1979 Gen. Rel. Grav. 11, 227

2. York J W 1989, in: Frontiers in Numerical Relativity, eds. C P Evans, L S Finn (Cambridge: Cambridge University Press)

3. Isenberg J 1995 Class. Quantum Grav. 12, 2249

4. Beig R, Ó Murchadha N 1996 Class. Quantum Grav. 13, 739

5. Besse A L 1987 Einstein Manifolds (Berlin Heidelberg New York: Springer)

6. Wheeler J A 1962 Geometrodynamics (New York: Academic Press)

7. Chaljub-Simon A 1981 Gen. Rel. Grav. 14, 743

8. Schouten J 1921 Math. Z. 11, 58

9. Beig R 1997 in: Mathematics of Gravitation, ed. P T Chrusciel, Banach Center Publ. 41, 109 
10. Geroch R 1969 Commun. Math. Phys. 13, 180

11. Geroch R 1970 J. Math. Phys. 11, 1955

12. Beig R 1997 ESI (Erwin Schrödinger Institute for Mathematical Physics) preprint 507

13. Brill D, Lindquist R 1963 Phys. Rev. 131, 471

14. Giulini D 1996 Class. Quantum Grav. 7, 1271

15. York J W 1980 in: Essays in General Relativity, ed. F J Tipler (New York: Academic Press)

16. Brandt S, Brügmann B 1997 Phys. Rev. Lett. 78, 3606

17. Lee J M, Parker T H 1987 Bull. Am. Math. Soc. 17, 37

18. Misner C W 1960 Phys. Rev. 118, 1110

19. Misner C W 1963 Ann. Phys. 24, 102

20. Obata M 1971 J. Diff. Geom. 6, 237

21. Beig R, Ó Murchadha N 1996 Commun. Math. Phys. 176, 723

22. Bowen J M, York J W 1980 Phys. Rev. 21, 2047

23. Kulkarni A D, Shepley L C and York J W 1983 Phys. Lett. 86A, 228

24. Bowen J M, Rauber J and York JW 1984 Class. Quantum Grav. 1, 591 